faecal water metabolic profiles. The effect of ethnicity is likely to be a combination of genetics, diet and environment. Ethnicity therefore must be accounted for as a potential confounder in this type of analysis.

\section{PTH-114 THE IMPACT OF IBD RELATED FATIGUE ON HEALTH- RELATED QUALITY OF LIFE: A LITERATURE REVIEW}

${ }^{1}$ Shellie Radford*, 'Jordan McGing, ${ }^{2}$ Wladyslawa Czuber-Dochan, 'Gordon W Moran. ${ }^{1}$ Nottingham Digestive Diseases Centre - The University Of Nottingham, Nottingham, UK; ${ }^{2}$ Kings College London, Florence Nightingale Faculty of Nursing, midwifery and palliative care, London, UK

\subsection{6/gutjnl-2019-BSGAbstracts.173}

Introduction Fatigue is a frequently reported symptom of inflammatory bowel disease (IBD) by patients in active disease and in remission. Fatigue related to chronic conditions reduces health-related quality of life (HRQoL) but has not been systematically reviewed in IBD.

Methods Four databases (CINAHL, EMBASE, PsycINFO, Medline) were systematically searched (September 2018) utilising combined search terms related to 'Fatigue', 'IBD \& 'HRQoL'. Searches were restricted to 'human', 'adult', 'primary research' $\&$ 'English language'. A time limit of 5 years (2013-2018) was set, in order to include only the most up to date studies. The search yielded 76 publications decreasing to 53 after duplicate removal. 34 studies were removed at abstract. Reviewers read 19 full text papers of which 8 were removed as they were irrelevant to the study aim. Two authors independently screened studies, extracted data and conducted quality appraisal. All discrepancies were resolved by discussion. A narrative review synthesis was conducted to analyse the data.

Results 11 publications were reviewed, 5 qualitative and 6 cross-sectional surveys presented data from 2823 adults with IBD. Studies revealed experience of fatigue was significantly related to three HRQoL areas; symptom acceptance, psychosocial factors such as social isolation, and decreased physical activity. Patients reporting higher levels of IBD fatigue had lower levels of symptom acceptance in terms of 'management' and 'adjustment'. Higher anxiety and depression were strongly linked to higher fatigue and reduced HRQoL. Higher levels of social support were associated with higher HRQoL, reduced physical activity was linked to higher fatigue levels, lowering HRQoL, but also as an intervention to reduce fatigue and improve HRQoL. Seven studies were of high quality. Appraisal revealed methodological shortcomings in a number of studies. Most frequent limitations were the use of multiple, un-validated, measures, comparison of results without appropriate statistical adjustment, failure to specify the level of disease activity of participants and assessment of both fatigue and HRQoL using the same tool.

Conclusions Depressive symptoms, anxiety, poor symptom management \& lack of social support are associated with greater severity of fatigue in IBD. Results from this review support the application of a psychosocial or exercise intervention for fatigue management. Perceptions of control and social support were associated with lower fatigue level and higher HRQoL. Further research is required to explore psychosocial variable in relation to IBD fatigue, with use of validated fatigue and HRQoL measures; and clearer characterisation of disease activity.

\section{PTH-115 VARIATION IN ASSESSMENT OF OUTCOMES FOR IBD IN ROUTINE CLINICAL PRACTICE: AN ETHNOGRAPHIC STUDY}

Violeta Razanskaite*, Bridget Young, Paula Williamson, Keith Bodger. University of Liverpool, Liverpool, UK

\subsection{6/gutjnl-2019-BSGAbstracts.174}

Introduction Several global initiatives are seeking to standardise outcome assessment for IBD in clinical trials (Core Outcome Sets) and routine care (e.g. ICHOM). The UK IBD Biologics Audit found inconsistent use of clinical disease activity indices among UK clinicians. We aimed to explore variability in outcome assessment in routine practice, evaluating the range of individual outcomes elicited and extent of standardisation and quantification.

Methods Ethnographic observations of 52 IBD clinic consultations by 17 IBD clinicians (8 consultants, 7 IBD nurses and 2 trainees) across four acute hospitals in the North West region of England. Consultations were observed, audio-recorded,

Abstract PTH-115 Table 1 Ten most common outcomes elicited, n (\%)

\begin{tabular}{|c|c|c|c|c|c|}
\hline \multicolumn{2}{|c|}{ All IBD patients $n=52$} & \multicolumn{2}{|c|}{ Crohn's disease $n=29$} & \multicolumn{2}{|c|}{ Ulcerative colitis or IBDU $n=23$} \\
\hline General wellbeing & $52(100)$ & General well-being & $29(100)$ & General well-being & $23(100)$ \\
\hline Abdominal pain & $44(85)$ & Abdominal pain & $25(86)$ & Stool frequency & $21(91)$ \\
\hline Stool frequency & $41(89)^{*}$ & Stool frequency & $20(87)^{*}$ & Blood in stool & $19(83)$ \\
\hline Blood with stool & $35(67)$ & Stool consistency & $18(62)$ & Abdominal pain & $19(83)$ \\
\hline Stool consistency & $33(63)$ & Blood with stool & $16(55)$ & Stool consistency & $15(65)$ \\
\hline Body weight & $27(52)$ & Appetite & $16(55)$ & Mucus & $11(48)$ \\
\hline Appetite & $25(48)$ & Body weight & $16(55)$ & Body weight & $11(48)$ \\
\hline Extra-intestinal & $25(48)$ & Extra-intestinal & $16(55)$ & Extra-intestinal & $9(39)$ \\
\hline Mucus & $22(42)$ & Energy levels & $14(48)$ & Appetite & $9(39)$ \\
\hline Energy levels & $19(37)$ & Mucus & $11(38)$ & Urgency & $8(35)$ \\
\hline
\end{tabular}

*Patients with stoma excluded $(n=6)$ 